\title{
A Surface Fairing Method Based on Torus Tool Machining
}

\author{
Zhang Bochang, Xu Rufeng \\ School of Mechanical Engineering and Automation \\ Beijing University of Aeronautics and Astronautics \\ Beijing, China, 100191
}

\author{
Ning Tao, Chen Zhitong \\ School of Mechanical Engineering and Automation \\ Beijing University of Aeronautics and Astronautics \\ Beijing, China, 100191
}

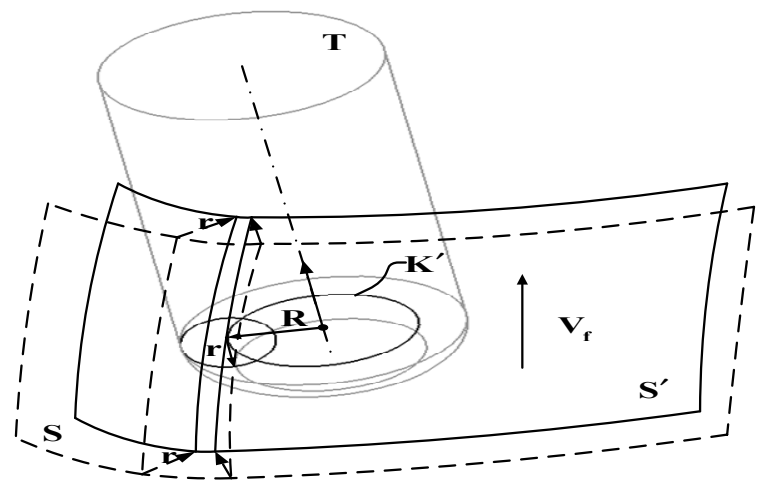

Figure 1. Equivalent relation between Tool and circle[7]

\section{INTRODUCTION}

In order to improve machining efficiency of the engine blade, many researchers studied tool position optimization algorithm of ring tool machining surface. This algorithm requires high surface smoothness [7].

According to the End-points error controlling method for tool position optimization in five-axis, which is put forward by the Jin Man[2], develop surface smoothness checked tools oriented to ring tool machining.

In this paper, take the data points as reference, which influence the row span and detected by the surface smoothness checked tools, extract iso-parametric lines including the data points, smoothening iso-parametric lines by using energy method. Re-fit surface again, checking row span, and repeating this progress, until Surface machining row-span is satisfied. At this time, it is considered that surface conform to the requirement of smoothing.

\section{DeVelopment Of Surface Smoothness ChecKed TOOLS}

\section{A. End-points error controlling method for tool position optimization in five-axis}

Figure 1 shows Torus Tool T, where circle radius is $\mathrm{R}$ 、 generating circle radius is $\mathrm{r}, \mathrm{S}$ represented surface machining, which is equivalent to Torus Tool $\mathrm{K}$ ', in which radius is R, Offset Surface S', whose distance to $S$ is r.

\section{B. Checked tools for surface smoothness}

According to end-points error controlling method for tool position optimization, calculate cutting path of surface $\mathrm{S}$, show in Fig2, LE0,LE1, $\cdots$ LEi-1 is drive line of next row tool-path from $1,2, \ldots$, i respectively, also the iso-

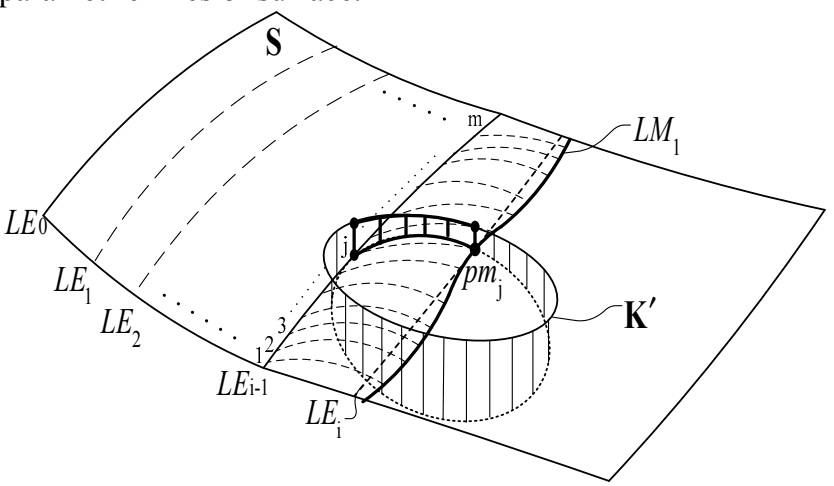

Figure 2. Schematic diagram of Checked Torus tools for surface smoothness

Calculate i-th row tool-path and take LEi-1 as drive line, in accordance with end-points error controlling method for tool position optimization, calculate the optimum cutting path corresponding to $\mathrm{m}$-th cutter location points respectively, connect the projection point pmi $(\mathrm{i}=1,2, \cdots, \mathrm{m})$, which are the optimum cutter location points projecting on the surface $\mathrm{S}$, and get the boundaries of parametric lines of surface. 
actual processing LM1, LEi is the iso-parametric lines cross point pmj.

Theory row span of i-th is $l=\left|L E_{i}-L E_{i-1}\right|$. But the machining region of tool actual processing is partial between LM1 and LEi-1. When calculate the i-th cutter location points, the drive line is LEi, not the LMi ,so repeating process is inevitable, and affecting machining efficiency greatly.

Analysis showed that the key factor influence the distance between LEi and LM1 is the point pmj that have the shortest distance from drive line LEi-1, with the increase of the distance from pmj and LEi-1, LEi will close to LM1, and the gap between them become smaller. Reaction on the surface is that fairness of surface contours cross pmj will be better. Therefore development of surface smoothness checked tools ,the basic idea is how and where to find pmj and surface contours.

The concrete implementation method:

Step1: Using end-points error controlling method for tool position optimization in nc machining, calculate the optimum cutting path respectively, obtain the projection point $\operatorname{pmi}(\mathrm{i}=1,2, \cdots, \mathrm{m})$, which are the optimum cutter location points corresponding to $\mathrm{m}$-th cutter location points in the i-row and projecting on the surface $\mathrm{S}$.

Step2: Find the point in $\operatorname{pmi}(\mathrm{i}=1,2, \cdots, \mathrm{m})$ that have the shortest distance from drive line LEi-1, makes Pmin=pmj;

Step3: extract iso-parametric lines LEi corresponding to Pmin , and it used as the surface contours to smooth processing.

\section{III. FAIRING SURFACE CONTOURS OF BLADE SURFACE}

\section{A. Determination of Objective Function}

Suppose parametric cubic B-spline curves interpolate in the given data points $\left\{P_{i}\right\} \quad(\mathrm{i}=0,1,2, \ldots, \mathrm{n})$, and it is:

$$
P_{0}(t)=\sum_{j=0}^{n+2} b_{j 0} N_{j, 3}(t)
$$

Where $b_{j 0} \quad(\mathrm{j}=0,1,2, \ldots, \mathrm{n}+2)$ is control vertex, $N_{j, 3}(t)$ is parametric cubic B-spline basis function, determined by the node vector $\left\{t_{i}\right\} \quad(\mathrm{i}=1,2, \ldots, \mathrm{n}+6)$, this paper adopt the precept of accumulating chordal parametrization

Our problem is seeking parametric cubic B-spline curves $P(t)$ defined on the node vector\{ $t_{i}$ \} $(\mathrm{i}=1,2, \ldots, \mathrm{n}+6)$,makes $P(t)$ have good fairness and modify data points within permissible error range. $P(t)$ can be represent as follows:

$$
P(t)=\sum_{j=0}^{n+2} b_{j} N_{j, 3}(t)
$$

take (3) second derivation:

$$
P^{\prime \prime}(t)=\sum_{j=0}^{n+2} b_{j} N_{j, 3}^{\prime \prime}(t)
$$

Where, $b_{j} \quad(\mathrm{j}=0,1, \ldots, \mathrm{n}+2)$ is Control Point of curve after fairness.

Requirement of fairness is that not only the small strain energy of curve but the even fluctuation of curvature after fairness. Based on this, we can get objective function as follows:

$$
F=\alpha \int k^{2} d s+\beta \sum_{i=1}^{n-1} d_{i}^{2}
$$

Where $d_{i}=\left|\frac{k_{i+1}-k_{i}}{l_{i+1}}-\frac{k_{i}-k_{i-1}}{l_{i}}\right|$ is called shear jerk in point $P_{i}$ of curve $P(t), l=\left\|P_{i}-P_{i-1}\right\|$ is chord length from $P_{i-1}$ to $P_{i}$ in curve, $k_{i}$ represent the relative curvature of $p_{i}=p\left(t_{i+3}\right) . \alpha, \beta$ are nonnegative constant fixed initially[8]. Smaller the value of $F$, the curve will be more fairing. Therefore, fairing method is under in the restrained conditions, makes the $\mathrm{F}$ reach its minimum value.

$$
\sum_{j=0}^{n+2}\left(b_{j}-b_{j 0}\right)^{2}<\varepsilon
$$

\section{B. Solution of Objective Function}

First of all, it can be transformed into unconstrained optimization problem, seeking control points $\left\{b_{j}\right\}$ $(\mathrm{j}=0,1,2, \ldots, \mathrm{n}+2)$

$$
G=\alpha \int k^{2} d s+\beta \sum_{i=1}^{n-1} d_{i}^{2}+\gamma \sum_{j=0}^{n+2}\left(b_{j}-b_{j 0}\right)^{2}
$$

Then taken the minimum. Second, adopt the precept of accumulating chordal parametrization, linear processing of (7) as: $d t$ instead of $d s, P^{\prime \prime}(t)^{2}$ instead of $k^{2}, D_{i}^{2}=\left[\frac{p^{\prime \prime}\left(t_{i+4}\right)-p^{\prime \prime}\left(t_{i+3}\right)}{l_{i+1}}-\frac{p^{\prime \prime}\left(t_{i+3}\right)-p^{\prime \prime}\left(t_{i+2}\right)}{l_{i}}\right]_{\text {replace }}^{2} d_{i}^{2}$.Then approximate representation of (7) is :

$$
G=\alpha \int_{0}^{t} p^{\prime \prime}(t)^{2} d t+\beta \sum_{i=1}^{n-1} D_{i}^{2}+\gamma \sum_{j=0}^{n+2}\left(b_{j}-b_{j 0}\right)^{2}
$$

By substitution of(3), (4) into the(8), solving the equation we deduce all control points $\left\{b_{j}\right\}$ $(j=0,1,2, \ldots, n+2)$ after fairing.

\section{SURFACE FAIRNESS}

Original data of blade is generally $M \times N$ data points(M is the munber of section lines, $\mathrm{N}$ is the number of data points in each optical section lines ). The fairing teps of blade surface as follows:

Step1: Deal with original data, fitting $M$ section line, and fairness them;

Step2: Design of blade surface based on Laying-off Law;

Step3:Find the smallest "dot" PRmin(i) in path intervals of the tool path depend on analysis tool for surface fairness, 
extract iso-parametric lines L(i)corresponding to PRmin(i), and it used as the surface contours to smooth processing;

Step4:Fairing surface contours L(i);

Step5: With original $M$ section line and Fairing surface contours L(i)construct a surface;

Repeating Step3、Step4、Step5, until path intervals is satisfied.

\section{EXAMPLES AND PRECISION ANALYSIS}

A blade have 10 section line, and 31 data points in each section line. Deal with original data, and smoothed section line. Comparing curvature between pre-and post fairing of blade section curve, show in Fig3, curvature of blade section curve fluctuate before fairing , curvature of blade section curve faired uniform variation, better than before fairing significantly. Fairing error is controlled within $0.05 \mathrm{~mm}$ error bounds. Fig4 shows comparing Light-Reflection between pre-and post fairing of blade surface.

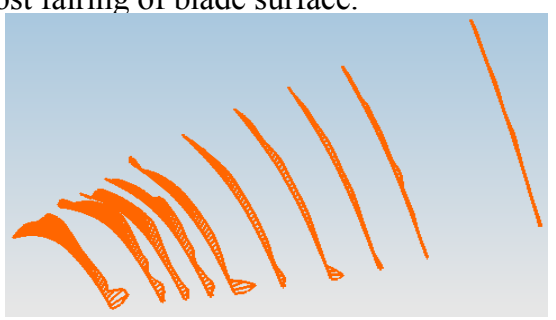

(a) Before fairing

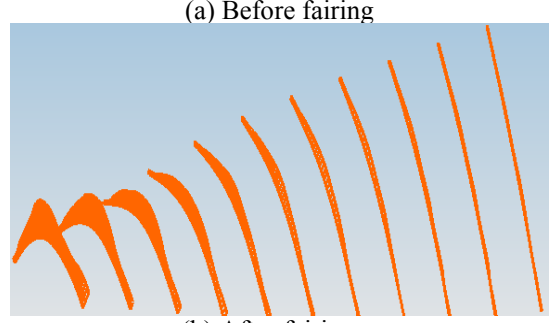

(b) After fairing

Figure 3. Comparing curvature between pre-and post fairing of blade section curve

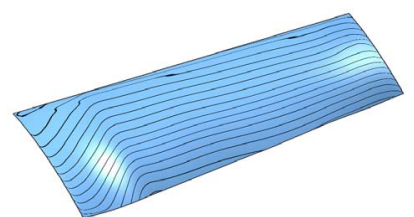

(a) Before fairing

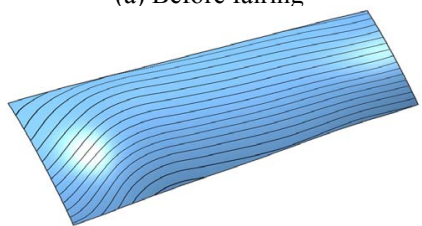

(b) After fairing

Figure 4. Comparing blade surface between pre-and post fairing

Find the imperfection, depend on analysis tool for surface fairness, extract surface iso-parametric lines corresponding to imperfection then fairing. With original section lines and these iso-parametric lines construct a surface; continue to find the imperfection. In this case, fairing once mainly contained a satisfactory path intervals. Experiment results as shown in Tab1 and Fig5. Experiments showed that tool path intervals lifting significantly.

TABLE I. TOOL PATH INTERVALS BETWEEN PRE-AND POST FAIRING

\begin{tabular}{|c|c|c|c|}
\hline $\begin{array}{c}\text { line number of tool } \\
\text { path }\end{array}$ & 1 & 2 & 3 \\
\hline Imperfection V & 0.005173 & 0.012155 & 0.019142 \\
\hline $\begin{array}{c}\text { path intervals before } \\
\text { fairing }\end{array}$ & 0.005173 & 0.006982 & 0.006987 \\
\hline $\begin{array}{c}\text { path intervals after } \\
\text { fairing }\end{array}$ & 0.02095 & 0.024171 & 0.024169 \\
\hline
\end{tabular}

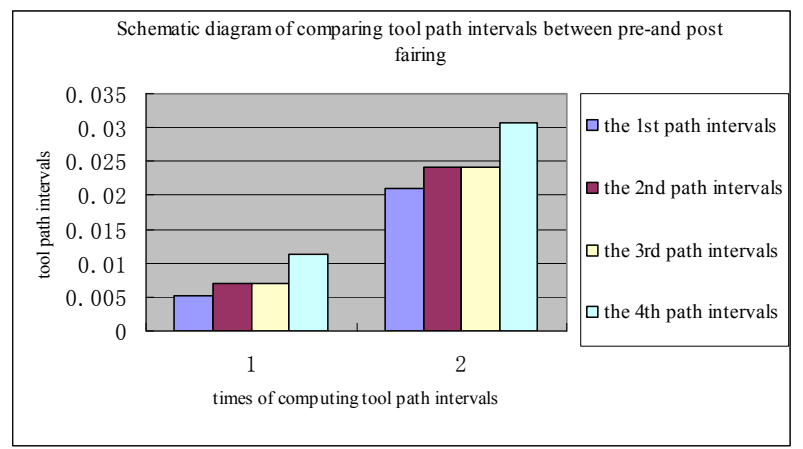

Figure 5. Schematic diagram of comparing tool path intervals between pre-and post fairing

\section{CONCLUSION}

This paper introduces end-points error controlling method for tool position optimization in 5-axis nc machining and fairing of cubic B-spline curves, development inspection tool for surface fairness based on two algorithms, fairing surface contours cross imperfection, in order to realize the target of the path intervals maximizing during machining. Practices show that this method increases the machining path intervals significantly, enhance the machining efficiency. Blade shape is different, or different parts of the same blade curvature difference, goal of maximum path intervals is corresponding different, the number of iterations should be different settings.

\section{ACKNOWLEDGEMENTS}

I would like to extend my sincere gratitude to my supervisor, Ning Tao, for his instructive advice and useful suggestions on my thesis. I also wish to thank Dr. Xu Rufeng, professor Chen Zhitong for their valuable discussions and encouragements.

This work supported by the Natural Science Foundation : Theoretical Research on free surface smooth design oriented to wide line machining of NC.

Grant No.51075021.

\section{REFERENCES}

[1] Tang Rongxi.CAD / CAM technology[M] Beijing University of Aeronautics and Astronautics Press, 1994 
[2] Jin Man Zhang Li Chen Zhitong. End-points error controlling method for torus tool position optimization in five-axis $\mathrm{NC}$ machining $[\mathrm{J}]$ Journal of Beijing University of Aeronautics and Astronautics. 2006, 32(9)

[3] Li Yunduan,Ning Tao.Research on N-side Region Surface Filling Algorithms [D] .Beijing University of Aeronautics and Astronautics,2007.02

[4] Li Yuan. CAGD technology\& application[M] Northwestern polytechnical university press

[5] Schneider R, Kobbelt L. Geometric fairing of irregular meshes for free-form surfacedesign. Computer-Aided Geometric Design, 2001, 18(4): 359 379.
[6] Xu Rufeng.Research on Tool Position Error Calculation and Tool Path Planning Methods in 5-axis Machining of Sculptured Surface[D] Beijing University of Aeronautics and Astronautics,2010.05

[7] Li Zhibing.Tool Position Optimization Technology for Cleaning-up Machining of Combined Surfaces'Root Area[D].Beijing University of Aeronautics and Astronautics,2010.04

[8] Mu Guowang, Tu Houjic. A GLOBAL FAIRING METHOD FOR CUBIC B-SPLINE CURVES JOURNAL OF ENGINEERING GRAPHICS[J]Journal of Engineering Graphics, 1998,1

[9] C. Castagnetti,E. Duc,and P. Ray.The Domain of Admissible Orientation concept: A new method for five-axis tool path optimisation[J] Computer-Aided Design ,2008,9. 40(9):938 950 